\title{
Numerical Nanofluid Simulation with Finite Volume Lattice-Boltzmann Enhanced Approach
}

\author{
J. Ghasemi ${ }^{1 \dagger}$, S. E. Razavi ${ }^{2}$ \\ ${ }^{1}$ Faculty of Engineering, University of Zanjan, Zanjan, 45195-313, Iran \\ ${ }^{2}$ School of Mechanical Engineering, University of Tabriz, Tabriz, Iran \\ †Corresponding Author Email:j.ghasemi@znu.ac.ir,jghasemi@tabrizu.ac.ir
}

(Received March 31, 2012; accepted October 31, 2012)

\begin{abstract}
In this paper, the finite volume Lattice-Boltzmann method is used to model the thermo-fluid behavior of nanofluid, in which nanoparticles are dispersed. The major internal and external forces including Brownian, repulsion and attracting DLVO, drag and buoyancy acting on nanoparticles are taken into account. All these forces make the thermal and dynamic mechanism inside the nanofluid improved. These models are established to simulate and enhance the heat transfer properties of nanoparticles in the $\mathrm{CuO}-\mathrm{H} 2 \mathrm{O}$ nanofluid as a test case. Also, convective heat transfer coefficient of the nanofluid is computed in different Reynolds numbers. The numerical approach is based on a modified and robust finite volume method.
\end{abstract}

Keywords: Finite volume lattice-Boltzmann, nanofluid, Brownian force, Convective heat transfer, Pressure-based flux factor, Energy distribution function.

\section{NOMENCLATURE}

$\begin{array}{cl}a & \text { particle acceleration } \\ \mathrm{A}_{\mathrm{H}} & \text { Hamaker coefficient } \\ \mathrm{D}_{\mathrm{n}} & \text { particle diameter } \\ \mathrm{F} & \text { force on a particle } \\ F_{H} & \text { gravitational force } \\ F_{D} & \text { drag force, } \\ F_{B} & \text { brownian force } \\ F_{D L V O} & \text { DLVO potential force } \\ \mathrm{f} & \text { particles distribution functions } \\ \mathrm{f}^{\mathrm{eq}} & \text { equilibrium of particle distribution function } \\ \mathrm{g} & \text { energy distribution functions } \\ g^{e q} & \text { equilibrium of energy distribution function } \\ \mathrm{k}_{\mathrm{bf}} & \text { base fluid conductivity } \\ \mathrm{K}_{\mathrm{p}} & \text { particle conductivity } \\ m_{p} & \text { particle mass }\end{array}$

\section{INTRODUCTION}

The recent technological developments as well as the industrial process intensification have made the need for more efficient heat exchanging systems a contemporary demand. The liquid coolants are widely used for heat transfer enhancement of electronic devices and heat exchangers. So, many efforts for dispersing small particles with high thermal conductivity in the

$\begin{array}{cl}P_{r} & \text { Prandtl number } \\ \mathbf{u} & \text { macroscopic velocity } \\ \mathrm{v}_{\mathrm{i}} & \text { particle microscopic velocity } \\ Z & \text { zeta potential } \\ \alpha & \text { thermal diffusivity, } \\ \varepsilon & \text { dielectric constant } \\ \rho & \text { macroscopic density } \\ \kappa_{D}^{-1} & \text { debye screening length } \\ \tau_{v} & \text { density relaxation time } \\ \tau_{T} & \text { energy relaxation time } \\ v & \text { kinematics viscosity } \\ \psi & \text { surface potentials } \\ \phi & \text { volumetric fraction of nanoparticles } \\ \text { DLVO } & \text { Derjaguin-Landau- Verwey -Overbeek } \\ \text { DDF } & \text { Double Distribution Function }\end{array}$

liquid coolant have been conducted to enhance thermal properties of the conventional fluids. The enhancement of thermal conductivity of fluids by suspending the solid particles of millimeter- or micrometer-sized suffers from number of drawbacks. The abrasive action of the particles causes erosion of components, clogging becomes a major problem in small flow passages and their requirement of momentum transfer increases the pressure drop considerably. The above bottleneck of 
slurries with micron or bigger size particles can be removed by using particles of nanometer dimensions. The concept was first materialised by series of research works at the Argonne National Laboratory and probably Choi (1995) was the first to call the fluids with particles of nanometer dimension suspended in them as "nanofluids" which has gained popularity. The heat transfer properties of a nanofluid strongly depend on the size, shape, and volume fraction of nanoparticles as well as on the type of the nanoparticles and base fluid (Duangthongsuk and Wongwises, 2009). In addition, the suspended nanoparticles distribution and temperature may play important roles in enhancing the heat transfer characteristics of nanofluids ( $\mathrm{Li}$ and Peterson, 2006; Chon and Kihm, 2005). Although the published papers on heat transfer enhancement by nanofluids are increasing, most of them are still limited to the macroscopic phenomenon inside the nanofluids. Since the volume fraction of nanoparticles is insufficient in comparison to base fluid, it is necessary to consider the heat transfer in nanofluids as two component fluid in microscopic treatments. However, in nanofluid as a colloidal fluid, the influences of external and internal forces, the suspended nanoparticles move in an irregular fashion. Main causes have been known, namely the Brownian, attraction and repulsion of DLVO, drag, and gravitational forces. Along with the main flow of nanofluids, behavior of suspended nanoparticles is irregular and all these factors must be applied in the thermo-hydrodynamic of nanofluid flow.

\section{NANOFLUID MODELING}

The conventional computational methods are not applicable to nanofluids as two component flow to reveal the nature of microscopic interactions and forces. Therefore, a microscopic or mesoscopic approach is needed to describe the interaction between the suspended nanoparticles and base fluid. Unlike the Navier-Stokes equations, the Lattice Boltzmann Method (LBM) possesses capability of incorporating microscopic interactions between nanoparticles and base fluid which can reveal the mesoscopic mechanism of thermo-hydrodynamics (Xuan and Yao, 2005; Yang and Lai, 2011). Therefore, this method seems to be suitable for handling the mentioned problems (Nemati et al., 2010; Cao et al., 1997). In finite difference LBMs, the Cartesian grid is used, which can be a shortcoming (Cao et al., 1997). To deal with complex domains, various proposals have been made to use boundary-fitted grids. During the past few years, some researchers attempted to use irregular lattices. Bouzidi et al. (2001) constructed a multi-relaxation LBM on a two-dimensional rectangular grid. Ubertini et al. (2005) developed a finite-volume scheme for the LBM on unstructured meshes in two dimensions, where complex boundary geometries could be handled. In this paper arbitrary quadrilateral cells are used by combining a cell-centered Finite-Volume and Lattice Boltzmann Methods (FV-LBM). The approach includes both the flow and heat transfer Boltzmann equations.

\subsection{Hydrodynamic FV-LBM Formulation of Nanofluid}

The nanofluid includes the suspension of nanoparticles and a base fluid, i.e., $\sigma=1,2$. Hence, the discretized integral form of lattice Boltzmann equation with BGK model for collision term in a nanofluid reads

$$
\begin{gathered}
\int_{\mathrm{S}}\left(\frac{\partial \mathrm{f}_{\mathrm{i}}^{\sigma}}{\partial \mathrm{t}}+\mathrm{v}_{\mathrm{i}} \cdot \nabla \mathrm{f}_{\mathrm{i}}^{\sigma}+\mathbf{a}_{\mathrm{i}}^{\sigma} \cdot \nabla_{\mathrm{v}_{\mathrm{i}}} \mathrm{f}_{\mathrm{i}}^{\sigma}+\right. \\
\left.\frac{1}{\tau_{v}}\left(\mathrm{f}_{\mathrm{i}}^{\sigma}-\mathrm{f}_{\mathrm{i}}^{\mathrm{eq}, \sigma}\right)\right) \mathrm{ds}=0 \\
\mathrm{i}=0,1, \ldots, \mathrm{M}
\end{gathered}
$$

where $\mathrm{v}_{\mathrm{i}}$ shows the particle microscopic velocity in the i-direction. The $\mathbf{a}=\mathbf{F} / m_{p}$ is acceleration caused by force $\mathbf{F}$ on a particle with mass, $m_{p}$ and $\mathrm{f}^{\mathrm{eq}}$ denotes the equilibrium of particles distribution functions. Also $\tau_{v}$ displays the density relaxation time which is related to the kinematic viscosity as.

$v=\left(\tau_{v}-\frac{1}{2}\right) c^{2} \delta t$

where $c=\sqrt{3 R T}$ is the lattice speed. For a Newtonian fluid, the kinematic viscosity $v$ is calculated by (Rahmati and Ashrafizaadeh, 2009):

$v=\frac{\left(2 \tau_{v}-1\right)}{6}$

For the two-dimensional problem, the well-known $\mathrm{D}_{2} \mathrm{Q}_{9}$ lattice is widely used. Also, microscopic velocities, $v_{i}$ are determined based on a generic form (Nemati et al,. 2010; Bouzidi et al., 2001). A cellcentered finite-volume on arbitrary cells is shown in Fig. 1. According to Fig. 1, the following approximations can be made

$$
\begin{aligned}
& \int_{\text {abcd }} \frac{\partial \mathrm{f}_{\mathrm{i}}}{\partial \mathrm{t}} \mathrm{ds} \approx\left[\frac{\partial \mathrm{f}_{\mathrm{i}}}{\partial \mathrm{t}}\right]_{\mathrm{I}, \mathrm{J}} \cdot \mathrm{S}_{\mathrm{I}, \mathrm{J}} \\
& \text { and } \\
& \left\{\begin{array}{l}
\int_{\text {abcd }} \mathrm{v}_{\mathrm{i}} \cdot \nabla \mathrm{f}_{\mathrm{i}} \mathrm{ds} \approx \sum_{\mathrm{k}} \mathrm{v}_{\mathrm{i}} \cdot \mathrm{N}_{\mathrm{k}}\left[\tilde{\mathrm{f}}_{\mathrm{i}}\right]_{\mathrm{k}} \\
\mathrm{N}_{\mathrm{k}}=\left(\Delta \mathrm{y} \mathbf{i}-\Delta \mathrm{x} \mathbf{j}_{\mathrm{k}}, \mathrm{k}=\mathrm{ab}, \mathrm{bc}, \mathrm{cd}, \mathrm{da}\right.
\end{array}\right.
\end{aligned}
$$

Also, we use the convective flux treatment of thermohydrodynamic lattice Boltzmann, which takes into account the second-order pressure- and temperaturebased biasing factors (Razavi et al., 2009; Ghasemi and Razavi, 2010). This makes the scheme to be dominantly upwind, broadens the numerical stability region, and improves accuracy for both the velocity and temperature fields and reduces the convergence steps.

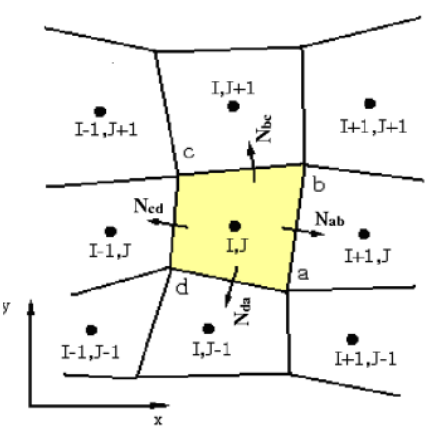

Fig. 1. Cell-centered finite volumes 
Here, the pressure-based biasing factor for convective fluxes is employed. A typical determination of this factor, $\boldsymbol{\xi}$ for side $\underline{\boldsymbol{a} \boldsymbol{b}}$ of cell $(\mathrm{I}, \mathrm{J})$ reads

$$
\begin{aligned}
& \xi_{\mathrm{ab}-\mathrm{L}}^{\sigma}=\frac{\mathrm{p}_{\mathrm{I}-1, \mathrm{~J}}}{\mathrm{p}_{\mathrm{I}-1, \mathrm{~J}}+\mathrm{p}_{\mathrm{I}, \mathrm{J}}}, \\
& \xi_{\mathrm{ab}-\mathrm{R}}^{\sigma}=\frac{\mathrm{p}_{\mathrm{I}, \mathrm{J}}}{\mathrm{p}_{\mathrm{I}, \mathrm{J}}+\mathrm{p}_{\mathrm{I}+1, \mathrm{~J}}}, \\
& \xi_{\mathrm{ab}}^{\sigma}=\frac{\xi_{\mathrm{ab}-\mathrm{L}}^{\sigma}+\xi_{\mathrm{ab}-\mathrm{R}}^{\sigma}}{2}
\end{aligned}
$$

Consequently, the convective fluxes are approximated as following:

$$
\begin{aligned}
& \int_{\mathrm{abcd}} \mathrm{v}_{\mathrm{i}} \cdot \nabla \mathrm{f}_{\mathrm{i}} \mathrm{ds} \approx \\
& \left\{\mathrm{v}_{\mathrm{i}} \cdot \mathrm{N}_{\mathrm{ab}}\left(\xi_{\mathrm{ab}}^{\sigma}\left[\mathrm{f}_{\mathrm{i}}\right]_{\mathrm{I}, \mathrm{J}}+\left(1-\xi_{\mathrm{ab}}^{\sigma}\right)\left[\mathrm{f}_{\mathrm{i}}\right]_{\mathrm{I}+1, \mathrm{~J}}\right)+\right. \\
& \mathrm{v}_{\mathrm{i}} \cdot \mathrm{N}_{\mathrm{bc}}\left(\xi_{\mathrm{bc}}^{\sigma}\left[\mathrm{f}_{\mathrm{i}}\right]_{\mathrm{I}, \mathrm{J}}+\left(1-\xi_{\mathrm{bc}}^{\sigma}\right)\left[\mathrm{f}_{\mathrm{i}}\right]_{\mathrm{I}, \mathrm{J}+1}\right)+ \\
& \mathrm{v}_{\mathrm{i}} \cdot \mathrm{N}_{\mathrm{cd}}\left(\xi_{\mathrm{cd}}^{\sigma}\left[\mathrm{f}_{\mathrm{i}}\right]_{\mathrm{I}, \mathrm{J}}+\left(1-\xi_{\mathrm{cd}}^{\sigma}\right)\left[\mathrm{f}_{\mathrm{i}}\right]_{\mathrm{I}-1, \mathrm{~J}}\right)+ \\
& \left.\mathrm{v}_{\mathrm{i}} \cdot \mathrm{N}_{\mathrm{da}}\left(\xi_{\mathrm{da}}^{\sigma}\left[\mathrm{f}_{\mathrm{i}}\right]_{\mathrm{I}, \mathrm{J}}+\left(1-\xi_{\mathrm{da}}^{\sigma}\right)\left[\mathrm{f}_{\mathrm{i}}\right]_{\mathrm{I}, \mathrm{J}-1}\right)\right\}
\end{aligned}
$$

Applying the pressure-biasing factor enabled the scheme to compensate some shortcomings, specially, the numerical instability of randomly behaving force term of nanofluid lattice Boltzmann equations. By linear approximation, the integration of the collision terms in Eq. (1) becomes

$$
\begin{aligned}
& -\int_{\text {abcd }} \frac{1}{\tau_{\sigma}}\left(\mathrm{f}_{\mathrm{i}}-\mathrm{f}_{\mathrm{i}}^{\mathrm{eq}}\right) \mathrm{ds}= \\
& -\frac{\mathrm{S}_{\mathrm{I}, \mathrm{J}}}{\tau_{\sigma}}\left[\frac{1}{4}\left[\Delta \mathrm{f}_{\mathrm{i}}\right]_{\mathrm{I}, \mathrm{J}}+\right. \\
& \frac{1}{8}\left\{\left[\Delta \mathrm{f}_{\mathrm{i}}\right]_{\mathrm{I}+1, \mathrm{~J}}+\left[\Delta \mathrm{f}_{\mathrm{i}}\right]_{\mathrm{I}, \mathrm{J}+1}+\left[\Delta \mathrm{f}_{\mathrm{i}}\right]_{\mathrm{I}-1, \mathrm{~J}}+\right. \\
& \left.\left[\Delta \mathrm{f}_{\mathrm{i}}\right]_{\mathrm{I}, \mathrm{J}-1}\right\}+\frac{1}{16}\left\{\left[\Delta \mathrm{f}_{\mathrm{i}}\right]_{\mathrm{I}+1, \mathrm{~J}-1}+\right. \\
& \left.\left.\left[\Delta \mathrm{f}_{\mathrm{i}}\right]_{\mathrm{I}+1, \mathrm{~J}+1}+\left[\Delta \mathrm{f}_{\mathrm{i}}\right]_{\mathrm{I}-1, \mathrm{~J}+1}+\left[\Delta \mathrm{f}_{\mathrm{i}}\right]_{\mathrm{I}-1, \mathrm{~J}-1}\right\}\right] ; \\
& \Delta \mathrm{f}_{\mathrm{i}}=\mathrm{f}_{\mathrm{i}}-\mathrm{f}_{\mathrm{i}}^{\mathrm{eq}}
\end{aligned}
$$

The $\mathrm{f}_{\mathrm{i}}$ 's are updated by a modified fifth-order RungeKutta scheme (Ghasemi and Razavi, 2010). The particles equilibrium function is given by Peng et al. (2003):

$$
\begin{aligned}
& \mathrm{f}_{0}^{\mathrm{eq}}=\frac{4}{9} \rho\left[1-\frac{3}{2 \mathrm{c}^{2}} \mathbf{u} \cdot \mathbf{u}\right] ; \\
& \mathrm{f}_{1,2,3,4}^{\mathrm{eq}}=\frac{1}{9} \rho\left[1+\frac{3}{\mathrm{c}^{2}} \mathrm{v}_{\mathrm{i}} \cdot \mathbf{u}+\frac{9}{2 \mathrm{c}^{4}}\left(\mathrm{v}_{\mathrm{i}} \cdot \mathbf{u}\right)^{2}-\frac{3}{2 \mathrm{c}^{2}} \mathbf{u} \cdot \mathbf{u}\right] \\
& \mathrm{f}_{5,6,7,8}^{\mathrm{eq}}=\frac{1}{36} \rho\left[1+\frac{3}{\mathrm{c}^{2}} \mathrm{v}_{\mathrm{i}} \cdot \mathbf{u}+\frac{9}{2 \mathrm{c}^{4}}\left(\mathrm{v}_{\mathrm{i}} \cdot \mathbf{u}\right)^{2}-\frac{3}{2 \mathrm{c}^{2}} \mathbf{u} \cdot \mathbf{u}\right]
\end{aligned}
$$

In Eq. (1), third term shows the force which is important for the action of base fluid on the nanoparticles. Since the volumetric fraction of nanoparticles is very small compared to the base fluid, therefore, it is ignored. In practice, due to the complexity of numerical calculations, reducing the computing time and accelerating the convergence, a part of this force as an additional term is included in the collision and the other part is inserted in the equilibrium distribution function. Hence, one can write

$$
\begin{gathered}
\left(\frac{\partial f}{\partial t}\right)_{c}=-\frac{1}{\tau_{v}}\left(f(\mathbf{x}, t)-\bar{f}^{e q}(\mathbf{x}, t)\right)+ \\
\frac{2 \tau_{v}-1}{2 \tau_{v}} \frac{D}{M c^{2}} \mathbf{v . F}
\end{gathered}
$$

In Eq. (10), D shows the dimension and the following relation is established

$\bar{f}^{e q}=f^{e q}\left(\rho, \mathbf{u}+\frac{1}{2 \rho} \mathbf{F}\right)$

This scheme is of second-order accuracy having better stability compared to the others (Buick and Greated, 1999).

\subsection{Main Forces Acting on Nanoparticles in a Nanofluid}

Similar to a colloidal fluid, the nanoparticles in nanofluid are under the influence of buoyancy, gravitational, drag, and Brownian forces. Also, the aggregation and dispersion forces determined resulting from the attractive and repulsive potential based on the DLVO theory. For the buoyancy, $F_{H}$, due to gravitational force one would write

$F_{H}=\frac{4}{3} \pi a^{3} g \Delta \rho$

where $a$ and $\Delta \rho$ are radius of nanoparticles and density difference of nanoparticles with base fluid respectively. The drag force, $F_{D}$, is expressed as:

$F_{D}=-6 \pi \mu a . \Delta u$

where $\mu$ shows the viscosity of base fluid and $\Delta u$ the velocity difference between the particle and fluid. Keblinski et al. (2002) estimated effect of Brownian motion in conductive heat transfer of nanofluids according to the Einstein theory. This theory demonstrates that whatever the particles be tiny, the diffusion time for them would be shorter compared to the heat diffusion time, and the Brownian motion becomes more observable. The Brownian force, $F_{B}$, on a particle, $\boldsymbol{i}$, is shown as (Nemati et al., 2010; Xuan et al., 2005)

$F_{B}^{i}=C_{B}^{i} \frac{k_{B} T\left(r_{i}\right)}{a}$

where $C_{B}^{i}$ and $k_{B}$ denote the compatibility factor and Boltzmann coefficient respectively. When a nanoparticle is placed in the polarized solution, depending on the chemical properties of particle and base fluid, due to solution ionization, the ion bonding or exchanging the particle surface becomes charged which produces electrostatic repulsion and Van der Waals attraction forces. Regarding this theory, electrostatic repulsion is formed because of the generation of electric potential. This potential is produced due to the formation of the charged dual layer caused by the fluid. The repulsion potential for two particles having the radii $a_{1}, a_{2}$ and surface potentials $\psi_{s 1}, \psi_{s 2}$ is expressed as (Duncan and Linavre, 1997) 


$$
\begin{aligned}
& \psi_{R}(r, T)=\frac{\pi \varepsilon a_{1} a_{2}\left(\psi_{s 1}^{2}+\psi_{s 2}^{2}\right)}{\left(a_{1}+a_{2}\right)} \mathbf{x} \\
& \left\{\frac{2 \psi_{s 1} \psi_{s 2}}{\left(\psi_{s 1}^{2}+\psi_{s 2}^{2}\right)} \operatorname{Ln}\left(\frac{1+e^{-\kappa_{D}(r-2 a)}}{1-e^{-\kappa_{D}(r-2 a)}}\right)\right. \\
& \left.+\operatorname{Ln}\left(1-e^{-2 \kappa_{D}(r-2 a)}\right)\right\}
\end{aligned}
$$

By equating the radii $\left(a_{1}=a_{2}=a\right)$ and surface potentials $\left(\psi_{s 1}=\psi_{s 2}=\psi_{s}\right)$ for two neighboring particles, one obtains

$$
\Psi_{R}(r, T)=\frac{\pi \varepsilon a \psi_{s}^{2}}{2} \operatorname{Ln}\left(1-e^{-\kappa_{D}(r-2 a)}\right), r>2 a
$$

In (Eq. (16), $\varepsilon$ denotes the dielectric constant of base fluid, and $\psi_{s}$ the particle surface potential as following:

$$
\psi_{s}=\frac{Z e}{\varepsilon\left(a+a^{2} \kappa_{D}\right)}
$$

In Eq. (17), $Z e$ shows the nanoparticles charge ( $Z$ is called the Zeta potential), and $\kappa_{D}^{-1}$ denotes the Debye screening length. Hence, one gets

$\kappa_{D}^{-1}=\sqrt{\frac{\varepsilon k_{B} T(1-\phi)}{e^{2}\left(\rho_{i}+n Z\right)}}$

In Eq. (18), the presence of nanoparticles has been appeared by $(1-\phi)$, where $\phi$ presents the volumetric fraction of nanoparticles. Also, $\rho_{i}$ denotes the ions crowd. The other considerable force is the Van der Waals attraction. According to the DLVO theory, this attraction potential is determined as,

$\psi_{A}=-\frac{1}{12} A_{H}\left[\frac{D_{n}^{2}}{r^{2}-D_{n}^{2}}+\frac{D_{n}^{2}}{r^{2}}+2 \operatorname{Ln}\left(1-\frac{D_{n}^{2}}{r^{2}}\right)\right]$

where, $D_{n}$ and $A_{H}$ show the particle diameter and Hamaker coefficient. Here, $A_{H}$ is independent of particle geometry and being a function of physical properties of nanoparticles and base fluid. Hence, the total DLVO potential reads,

$\Psi(r, T)=\Psi_{R}(r, T)+\Psi_{A}(r, T)$

The resulting force from this potential is determined by,

$$
F_{D L V O}=\frac{\partial \Psi(r, T)}{\partial r}
$$

Thence, vector summation of mentioned forces exerting on $\mathrm{n}$ nanoparticles in unit volume becomes:

$$
\mathbf{F}=\frac{n \cdot\left(F_{D L V O}+F_{B}+F_{D}+F_{H}\right)}{V o l .}
$$

Actually, the volume fraction of nanoparticles is negligible in comparison to the base fluid. Then the internal force of nanoparticles to base fluid is vanished.
The macroscopic density $\rho$, and velocity, $\mathbf{u}$ are determined as

$\rho=\sum_{\mathrm{i}=0}^{8} \mathrm{f}_{\mathrm{i}}, \quad \rho \mathbf{u}=\sum_{\mathrm{i}=0}^{8} \mathrm{v}_{\mathrm{i}} \mathrm{f}_{\mathrm{i}}$

\subsection{Thermal FV-LBM Formulation of Nanofluid}

The existing lattice Boltzmann models for thermal flows in the literature fall into three categories, i.e., the passive scalar, the multi-speed, and Double Distribution Function (DDF) approaches. The passive-scalar approach utilizes the fact that the macroscopic temperature satisfies the same evolution equation as a passive scalar if the viscous heat dissipation and compression work done by the pressure are negligible (Massaioli et al., 1993; He et al., 1998). The multispeed models are build up of the Lattice BhatnagarGross-Krook (LBGK) models for isothermal flows. The main disadvantage of the multi-speed LBGK models is that they suffer from numerical instability and are suited for problems with restricted temperature ranges (Shi et al., 2004). In addition, the multi-speed LBGK models using a single relaxation time are limited to problems with fixed Prandtl numbers, which have less engineering application. The DDF applies an additional distribution function as energy distribution to describe the evolution of temperature field (Palmer et al., 2000). The DDF holds better numerical stability and engineering applications. Hence, it has been used in present work. Considering the fact that the nanoparticles energy obeys the Boltzmann distribution and assuming the BGK approximation, thermal Boltzmann equation for base fluid $(\sigma=1)$ and nanoparticles $(\sigma=2)$ is stated as,

$$
\begin{aligned}
& \int_{\mathrm{s}}\left(\frac{\partial \mathrm{g}_{\mathrm{i}}^{\sigma}}{\partial \mathrm{t}}+\mathrm{v}_{\mathrm{i}} \cdot \nabla \mathrm{g}_{\mathrm{i}}^{\sigma}+\right. \\
& \left.\frac{1}{\tau_{\mathrm{T}}}\left(\mathrm{g}_{\mathrm{i}}^{\sigma}-\mathrm{g}_{\mathrm{i}}^{\mathrm{eq}, \sigma}\right)-\Theta\right) \mathrm{ds}=0, \\
& \mathrm{i}=0,1, \ldots, \mathrm{M}
\end{aligned}
$$

where $\tau_{T}$ shows the energy relaxation time which is related to the thermal diffusion coefficient as

$\alpha_{T}=\left(\tau_{T}-\frac{1}{2}\right) c^{2} \delta t$

For a fluid with low Prandtl number (or low Eckert number) the term $\Theta$ could be neglected (Van et al., 1997). In this case the Prandtl number is defined as,

$\operatorname{Pr}=\frac{v}{\alpha}=\frac{2 \tau_{v}-1}{2 \tau_{T}-1}$

The discretization of Eq. (24) in two dimensions on a $D_{2} Q_{9}$ lattice is similar to Eq. (1). Where in Eq. (6) the pressure is replaced by temperature (Ghasemi and Razavi, 2010). The equilibrium energy distribution function, $g^{e q}$, is expressed as following 


$$
\begin{aligned}
& \mathrm{g}_{0}^{\mathrm{eq}}=-\frac{2 \rho \varepsilon}{3} \frac{\mathbf{u}^{2}}{\mathrm{c}^{2}} \\
& \mathrm{~g}_{1,2,3,4}^{\mathrm{eq}}=\frac{\rho \varepsilon}{9}\left[\frac{3}{2}+\frac{3}{2 \mathrm{c}^{2}} \mathrm{v}_{\mathrm{i}} \cdot \mathbf{u}+\frac{9}{2 \mathrm{c}^{4}}\left(\mathrm{v}_{\mathrm{i}} \cdot \mathbf{u}\right)^{2}-\frac{3}{2 \mathrm{c}^{2}} \mathbf{u} \mathbf{u}\right] \\
& \mathrm{g}_{5,6,7,8}^{\mathrm{eq}}=\frac{\rho \varepsilon}{36}\left[3+\frac{6}{\mathrm{c}^{2}} \mathrm{v}_{\mathrm{i}} \cdot \mathbf{u}+\frac{9}{2 \mathrm{c}^{4}}\left(\mathrm{v}_{\mathrm{i}} \cdot \mathbf{u}\right)^{2}-\frac{3}{2 \mathrm{c}^{2}} \mathbf{u} \mathbf{u}\right]
\end{aligned}
$$

where $\varepsilon=\mathrm{DRT} / 2$ and,

$$
\rho \varepsilon=\sum_{\mathrm{i}=0}^{8} \mathrm{~g}_{\mathrm{i}}
$$

The Chapman-Enskog expansion for density distribution function can recover the continuity, Navier-Stokes, and energy equations. The detailed derivation can be found in D'Orazio and Succi (2004), Succi (2001) and will not be shown here. The physical properties of nanofluid are determined as following

$$
\begin{aligned}
& \bar{\rho}^{\sigma}=\rho^{\sigma} / \sum_{\sigma=1}^{2} \rho^{\sigma}, \bar{v}=2 \sum_{\sigma=1}^{2} \bar{\rho}^{\sigma} \tau^{\sigma}-1 / 6, \\
& \overline{\mathbf{u}}=\sum_{\sigma=1}^{2} \frac{\rho^{\sigma} \mathbf{u}^{\sigma}}{\tau^{\sigma}} / \sum_{\sigma=1}^{2} \frac{\rho^{\sigma}}{\tau^{\sigma}}, \\
& \bar{T}(x, t)=\frac{\sum_{\sigma}^{\sigma} T^{\sigma}(x, t)}{\sum_{\sigma} \rho^{\sigma} C_{p}^{\sigma}}
\end{aligned}
$$

\section{VELOCITY AND THERMAL BOUNDARY CONDITIONS}

In order to transform thermo-hydrodynamic boundary conditions into the boundary conditions for the distribution functions, we employ additional $D_{2} Q_{9}$

lattices at the edge of each cell for inflow, outflow, and solid boundaries, as shown in Figs. 2(a), 2(b) and 2(c), respectively ( $\mathfrak{R}$ replaced by $f$ for velocity and by $g$ for thermal boundary condition).

A suitable velocity boundary condition has been applied for hydraulic Boltzmann equation (Razavi et al., 2009). For thermal boundary conditions, a consistent open and solid boundary treatment is also used. The unknown energy distribution population at the boundary cells is decomposed into its equilibrium and non-equilibrium parts. Then, the $\mathrm{g}_{\mathrm{i}}$ 's at the node of boundary cell can be determined (Guo et al., 2007)

$$
\begin{aligned}
& \mathrm{g}_{\mathrm{i}}\left(x_{b}\right)=\mathrm{g}_{\mathrm{i}}^{\mathrm{eq}}\left(x_{b}, \rho_{b}, \varepsilon_{b}\right)+ \\
& \quad\left[\mathrm{g}_{\mathrm{i}}\left(x_{f}\right)-\mathrm{g}_{\mathrm{i}}^{\mathrm{eq}}\left(x_{f}, \rho_{f}, \varepsilon_{f}\right)\right]
\end{aligned}
$$

where, subscripts $\mathbf{b}$ and $\mathbf{f}$ shows the boundary cell and the nearest neighboring cell to boundary cell, respectively.

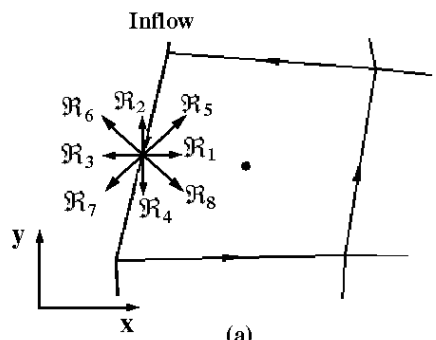

(a)

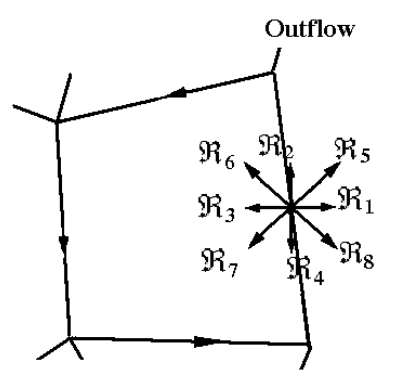

(b)

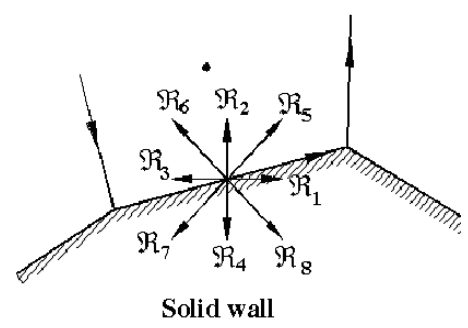

(c)

Fig. 2. Typical boundary cells (a) inflow, (b) outflow, (c) solid wall

A good approximation for unknown $\rho_{b}$ in boundary cells is $\rho_{f}$. For the Dirichlet type condition, the given temperature by energy distribution function, is applied directly on the boundary. The Neumann type condition is transferred to the Dirichlet type condition through the conventional second order finite difference approximation to obtain boundary temperature (Shu et al., 2002). Residuals, $\operatorname{Res}_{v}$ and $\operatorname{Res}_{\text {th }}$ of the velocity and temperature convergence criterion are set to

$$
\begin{aligned}
& \operatorname{Res}_{v}=\frac{\sum_{\mathrm{I}, \mathrm{J}}\left|\sqrt{\left(\mathrm{u}_{\mathrm{I}, \mathrm{J}}^{2}+\mathrm{v}_{\mathrm{I} . \mathrm{J}}^{2}\right)^{\mathrm{n}+1}}-\sqrt{\left(\mathrm{u}_{\mathrm{I}, \mathrm{J}}^{2}+\mathrm{v}_{\mathrm{I} . \mathrm{J}}^{2}\right)^{\mathrm{n}}}\right|}{\sum_{\mathrm{I}, \mathrm{J}}\left|\sqrt{\left(\mathrm{u}_{\mathrm{I}, \mathrm{J}}^{2}+\mathrm{v}_{\mathrm{I} . \mathrm{J}}^{2}\right)^{\mathrm{n}}}\right|} ; \\
& \operatorname{Res}_{\text {th }}=\frac{\sqrt{\sum_{I, J}\left|\mathrm{~T}_{I, J}^{\mathrm{n}+1}-\mathrm{T}_{\mathrm{I} . \mathrm{J}}^{\mathrm{n}}\right|^{2}}}{\sqrt{\sum_{\mathrm{I}, \mathrm{J}}\left|\mathrm{T}_{\mathrm{I} . \mathrm{J}}^{\mathrm{n}}\right|^{2}}}
\end{aligned}
$$

$\operatorname{Res}_{v}$ and $\operatorname{Res}_{\text {th }}$ are limited in numerical calculations to $5.0 \mathrm{E}-6$ and $1.0 \mathrm{E}-5$, respectively.

\section{NUMERICAL RESULTS}

For water as a base fluid one has $\rho_{i}=1.2044 \times 10^{20} \mathrm{~m}^{-3}$, $\mathrm{PH}=7$ at $300 \mathrm{k}$. Experimental measurements show 
that $10^{2}<Z<10^{3}$ in a typical nanofluid. For $\mathrm{CuO}$, $Z=457$ was taken. Due to the dilute crowd of nanoparticles $k_{p} \approx k_{b f}$ is considered. Also copper oxide nanoparticles read $\rho C=3.0 \times 10^{6} \mathrm{~J} / \mathrm{m}^{3} . k$ (Shukla and Dhir, 2008). Hence,

$\frac{n k_{B}^{2} T}{6 \mu \pi a}=4.833 \times 10^{-24} \frac{T}{a}$

$\phi \frac{\rho C k_{B} T}{6 \mu \pi a}=4.4 \times 10^{-15} \frac{\phi . T}{a}$

Having dielectric coefficient of water as $\varepsilon=8.9 \times 10^{-9} \mathrm{C}^{2} / \mathrm{N} \mathrm{m}^{2}$, therefore

$a \cdot \kappa_{D}=\left(\frac{3.175664 \times 10^{14} a^{2}}{(1-\phi) T}+\frac{6.7143 \times 10^{-3} \cdot \phi . Z}{a T(1-\phi)}\right)^{0.5}$

In Fig. 3, temperature variations, volumetric fraction, and nanoparticle sizes are plotted versus Debye screening length. The results reveal the loose dependence of temperature to Debye screening length where compared to the others. For $\mathrm{CuO}$ one has $A_{H}=18.1 \times 10^{-20} J$ (Linavre and Jordan, 2009).

Although the force convection in nanoparticles depends on Reynolds and Prandtl numbers, however, it is a function of several parameters as (Kakaç and Pramuanjaroenkij, 2009)

$N u_{N F}=f\left(\operatorname{Re}, \operatorname{Pr}, P e, k_{b f}, k_{p}, k n,\left(\rho c_{p}\right)_{p}\right.$,

$$
\phi \text {, particle shape, flow type) }
$$

By imposing the buoyant, drag, Brownian, and DLVO attraction and repulsion forces in thermo-hydrodynamic Boltzmann equation and employing the combined method in force and DDF for energy, the so-called equation has been solved. Extensive mesh testing was performed to guarantee a grid independent solution. The resulting force vectors exerting on the nanoparticles are shown in Fig. 4, for a twodimensional channel

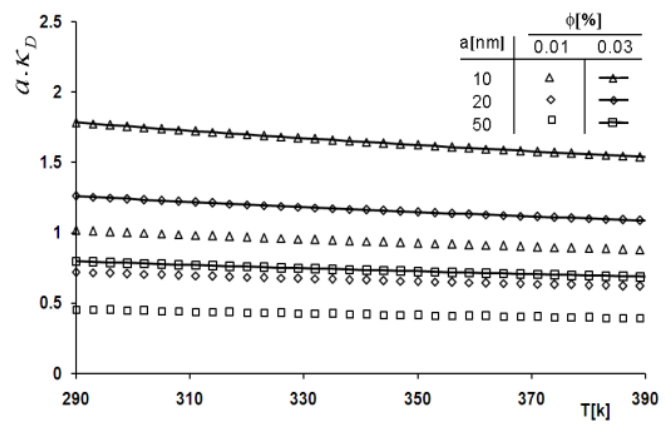

Fig. 3. Effect of temperature and nanoparticle volumetric fraction on Debye screening length

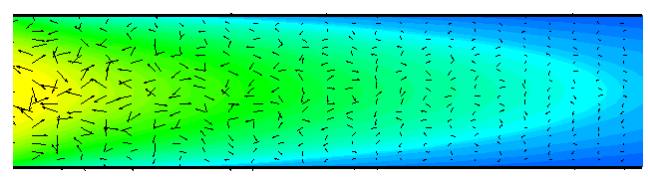

Fig. 4. Vectors of exerting forces on nanoparticles by base fluid, $\mathrm{a}=5 \mathrm{~nm} \cdot \phi=0.04$

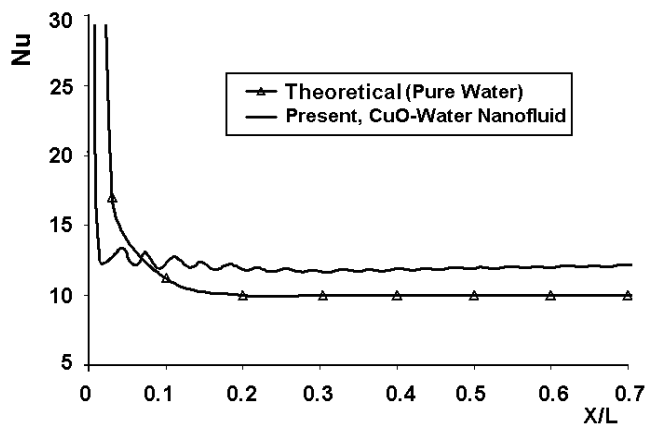

Fig. 5. The $\mathrm{Nu}$ variations on non-dimensional length of lower wall in $2 \mathrm{D}$ channel $\mathrm{Re}=73, \mathrm{Pr}=1.0$,

$$
\phi=0.01, a=5 \mathrm{~nm}
$$

Thus, the uniform inlet temperature is higher than the constant wall temperature. Fig. 4 depicts that this force is prevailing in high temperature regions. It is the origin of microscopic disturbances resulting from ballistic motion of nanoparticles which in turn enhances the heat transfer process. Fig. 5 presents the mean $\mathrm{Nu}$ variations along the lower channel wall for $\mathrm{CuO}$-water at $\mathrm{Re}=73$. It is observed from Fig. 5 that $\mathrm{Nu}$ of nanofluid undergoes small fluctuations along the wall. These fluctuations at the inlet higher temperature are due to the chaotic Brownian movements. Within the fullydeveloped region, the $\mathrm{Nu}$ of nanofluid is about $32 \%$ higher than the $\mathrm{Nu}$ of pure fluid, which is acceptable qualitatively in comparison to the results of other investigators (Al-Aswadi et al., 2010). The mean Nusselt variation versus volumetric fraction along the lower channel wall is observed in Fig. 6 for $\operatorname{Pr}=1.0$ at different Reynolds numbers. Here, the mean $\mathrm{Nu}$ experiences increment when the volumetric fraction of nanoparticles within the base fluid grows. Also, to validate the thermo-hydrodynamic numerical results firmly, a backward-facing step is utilized.

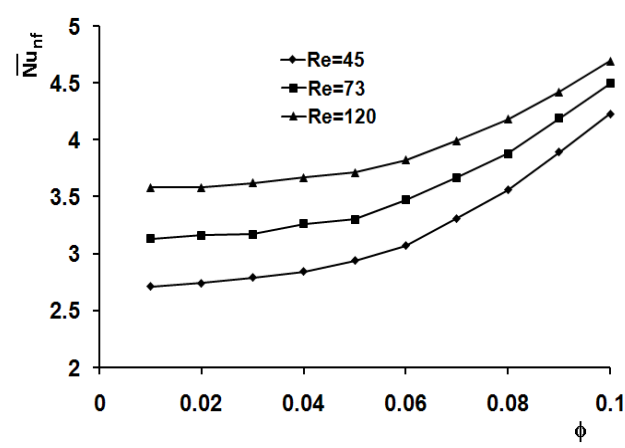

Fig. 6. Comparison of $\mathrm{Nu}$ versus nanoparticles volumetric fraction in various Reynolds numbers,

$$
a=5 \mathrm{~nm}
$$

Figure 7(a) shows the configuration and solution domain parameters. In the $\mathrm{x}$-direction, a fine grid was used in the regions near the point of reattachment to resolve the steep velocity gradients while a coarser grid was employed for downstream of that point. However, in the y-direction, a fine grid is generated near the top and bottom walls, also at the step. Figure 7(b) demonstrates a part of generated grids for expansion ratio $\mathrm{ER}=2(\mathrm{ER}=\mathrm{H} / \mathrm{s})$. Grid independence test is carried out for the nanofluid and it was found that a grid size of $798 \times 48$ ensures grid independent solution in this case. 
Similar to Aswadi et al. (2010), the step height and expansion ratio were fixed at $4.8 \mathrm{~mm}$ and 2 , respectively. The upstream wall $\left(\mathbf{L}_{\mathbf{U}}\right)$ and downstream wall ( $\mathbf{L}_{\mathbf{D}}$ ) were taken $50 \mathrm{~mm}$ and $1000 \mathrm{~mm}$, respectively.

The flow at the duct entrance was considered to be hydro-dynamically steady and fully developed. Figure 8(a) depicts the velocity distributions at different sections of downstream for $\mathrm{Re}=175$ with $5 \%$ volume fraction of $\mathrm{CuO}$-water nanofluid. Figures 8(b) to 8(d) show the distribution of velocity at four positions downstream the step. The results are in favorable agreement with other numerical solutions for the same conditions (Aswadi et al., 2010). Moreover, we compared the numerical heat transfer results of present study with other numerical solutions in backwardfacing step (Abu-Nada, 2008).

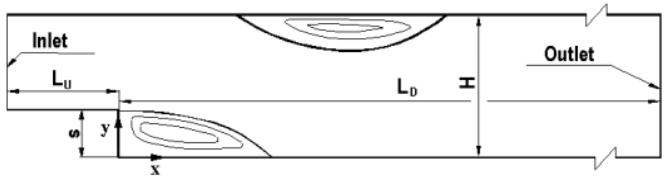

(a)

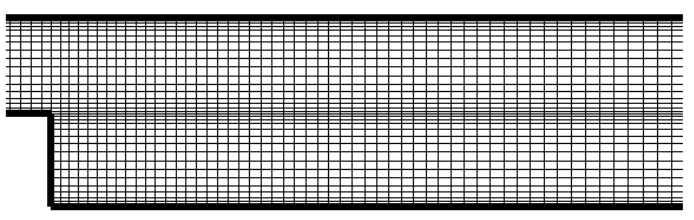

(b)

Fig. 7. (a) Schematic diagram for backward-facing step, (b) a part of generated grids.

Thus, the non-dimensional temperature defined as

$\theta=\frac{\mathrm{T}-\mathrm{T}_{\mathrm{c}}}{\mathrm{T}_{\mathrm{h}}-\mathrm{T}_{\mathrm{c}}}$

where $\mathbf{T}_{\mathbf{c}}, \mathbf{T}_{\mathbf{h}}$ are cold and hot wall temperatures, respectively. The boundary conditions are prescribed as follows:

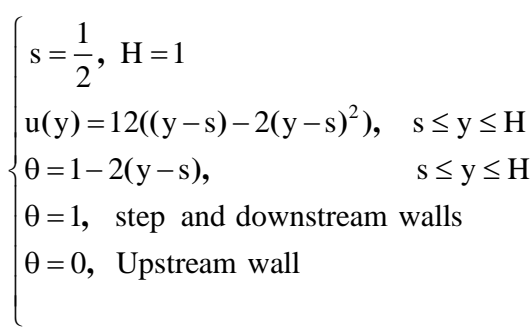

Fig. 9 presents the $\mathrm{Nu}$ variations along the bottom downstream step wall for $\mathrm{CuO}$-water. Where, $\operatorname{Re}=400, \operatorname{Pr}=1.0, \mathrm{a}=5 \mathrm{~nm}$ and $\phi=0.1$

This result reveals that the peak value of $\mathrm{Nu}$ is approximately located in reattachment point and the $\mathrm{Nu}$ of nanofluid is about $30 \%$ higher than the $\mathrm{Nu}$ of pure fluid. Moreover, the present solutions have reasonable agreement with the numerical results of Abu-Nada (2008).

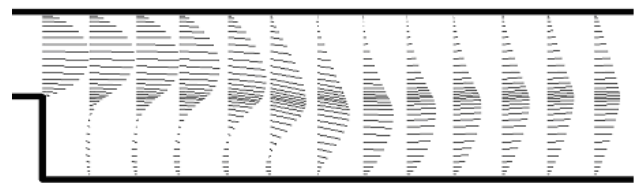

(a)

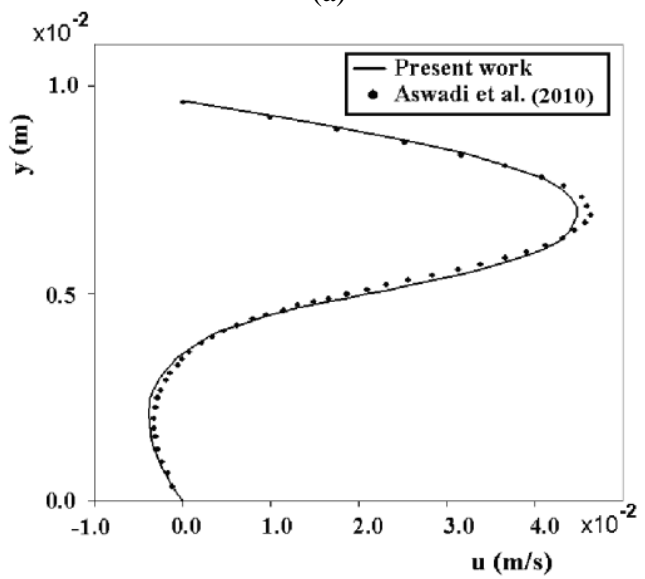

(b)

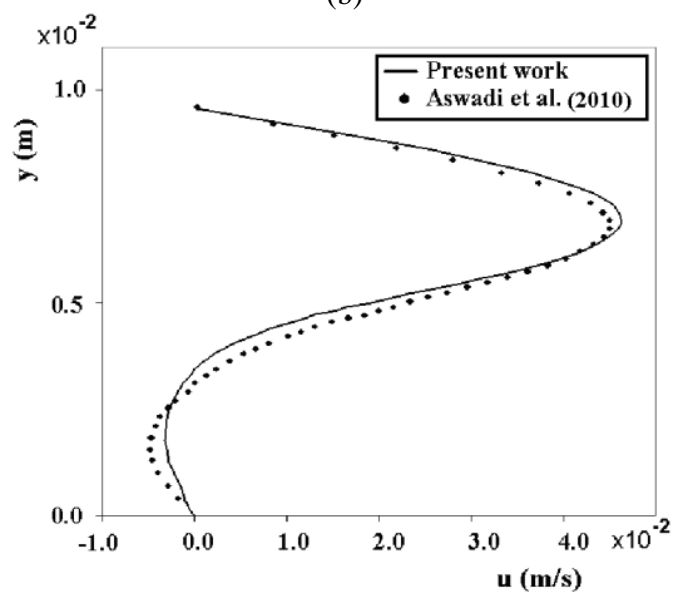

(c)

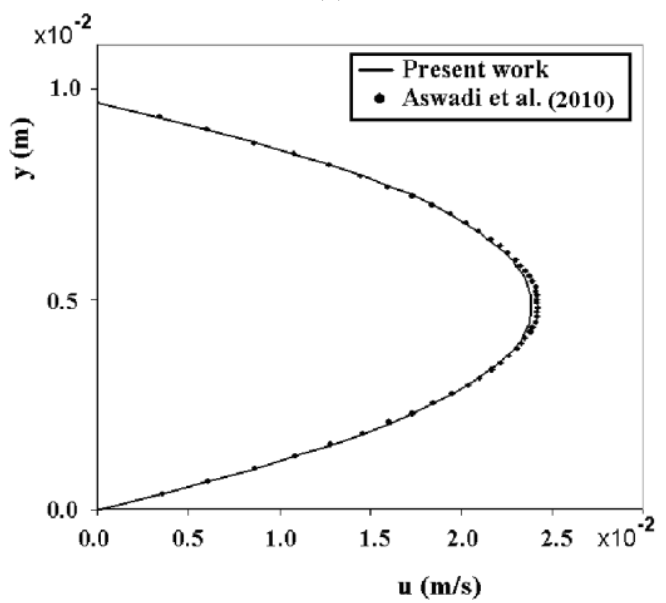

(d)

Fig. 8. Velocity distributions $(\mathrm{Re}=175)$ for $\mathrm{CuO}$-water nanofluid at different $\mathrm{x} / \mathrm{s}$, (a) Velocity profiles (b) $\mathrm{x} / \mathrm{s}=1.04$, (b) $\mathrm{x} / \mathrm{s}=1.92$, (c) $\mathrm{x} / \mathrm{s}=2.6$, (d) $\mathrm{x} / \mathrm{s}=32.8$. 


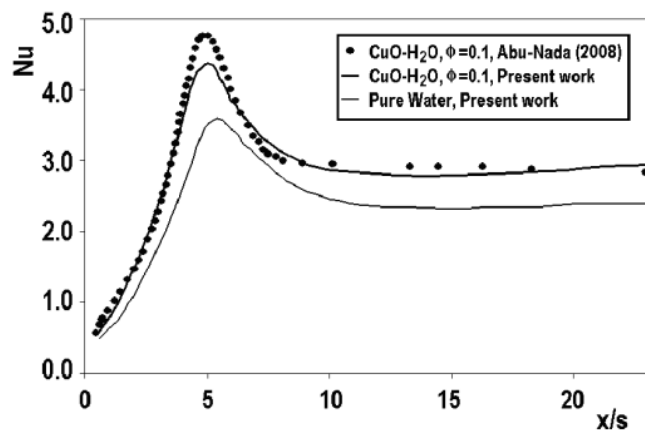

Fig. 9. The $\mathrm{Nu}$ variations on bottom downstream wall of backward-facing step.

\section{Conclusions}

In this paper the $\mathrm{D}_{2} \mathrm{Q}_{9}$ lattice along with non-uniform grids is used to simulate the thermo-fluid of nanofluid by combining a cell-centered FVM and DDF of thermal lattice Boltzmann method. The Brownian, repulsion and attracting DLVO, drag, and buoyancy forces acting on nanoparticles are considered for modeling the convective heat transfer and nanoparticles behavior in $\mathrm{CuO}-\mathrm{H} 2 \mathrm{O}$ nanofluid. In 2D channel within the fullydeveloped region, the present model determined that the $\mathrm{Nu}$ of nanofluid is about $32 \%$ higher than the $\mathrm{Nu}$ of pure fluid as reported in other researches. Moreover, this model enabled us to analyze the enhanced heat transfer of nanofluid for various practical $\mathrm{Re}$ and $\mathrm{Pr}$ numbers which is limited in classic lattice Boltzmann, especially for flow with heat transfer. This enhancement of LBM combined with FVM manifests itself by high numerical stability of nanofluid thermo-hydrodynamic in backward-facing step in presence of randomly Brownian force thermal lattice Boltzmann equations.

In general, the distinguished features of our work in comparison to the related previous investigations can be summarized as:

a) Utilizing the pressure- and temperature-based 2ndorder convective fluxes.

b) Considering the ballistic motion affected by randomly Brownian forces.

c) Applying DDF combined with 2nd-order accuracy of force term in LB equation and broadening the numerical stability.

\section{REFERENCES}

Abu-Nada, E., (2008). Application of nanofluids for heat transfer enhancement of separated flows encountered in a backward facing step, International Journal of Heat and Fluid Flow, 29(1), 242-249.

Al-Aswadi, A.A., H.A. Mohammed, N.H. Shuaib, A. Campo (2010). Laminar forced convection flow over a backward facing step using nanofluids. International Communications in Heat and Mass Transfer, 37(8), 950-957.
Bouzidi, M., D. d_Humieres, P. Lallemand, and L. S. Luo (2001). Lattice Boltzmann equation on a twodimensional rectangular grid, Journal of Computational Physics, 172, 704-717.

Buick, J. M., C.A. Greated (1999). Gravity in a Lattice Boltzmann model. Physical Review E., 61, 53075320 .

Cao, N., S. Chen, S. Jin, and D. Martinez (1997). Physical symmetry and lattice symmetry in the lattice Boltzmann method. Physical Review E., 55 (1), 21-24.

Choi, U. S. and J. A. Eastman (1995). Enhancing Thermal Conductivity of Fluids with Nanoparticles. ASME International Mechanical Engineering Congress and Exposition, Nov. 12-27, San Francisco, CA.

Chon, C.H., K.D. Kihm (2005). Thermal conductivity enhancement of nanofluids by Brownian motion. Journal of Heat Transfer, 127, 810.

D'Orazio, A. and S. Succi (2004). Simulating twodimensional thermal channel flows by means of a lattice Boltzmann method with new boundary conditions. Future Generation Computer Systems, 20, 935-944.

Duangthongsuk, W. and S. Wongwises (2009). Heat transfer enhancement and pressure drop characteristics of $\mathrm{TiO} 2-$ water nanofluid in a double-tube counter flow heat exchanger. International Journal of Heat and Mass Transfer, 52, 2059-2067.

Duncan, J. S. and H. Linavre (1997). Introduction to colloid and surface chemistry. Oxford, Jordan Hill.

Ghasemi, J. and S. E. Razavi (2010). On the finitevolume Lattice Boltzmann modeling of thermohydrodynamics. Computers and Mathematics with Applications, 60(5), 1135-1144.

Guo, Z. L., C. Zheng and B. Shi (2007). Thermal lattice Boltzmann equation for low Mach number flows: Decoupling model. Phys. Rev., 75(3).

He, X., S. Chen and G. D. Doolen (1998). A novel thermal model for the lattice Boltzmann method in incompressible limit. Journal of Computational Physics, 146, 282-300. 
Kakaç, S. and A. Pramuanjaroenkij (2009). Review of convective heat transfer enhancement with nanofluids. International, Journal of Heat and Mass Transfer, 52, 3187-319.

Keblinski, P. S., R. Phillpot, , S.U.S. Choi, J.A. Eastman (2002). Mechanisms of heat flow in suspensions of nano-sized particles (nanofluids). International Journal of Heat and Mass Transfer, $45,855-863$.

Li, C.H. and G.P. Peterson (2006). Experimental investigation of temperature and volume fraction variations on the effective thermal conductivity of nanoparticle suspensions (nanofluids). Journal of Appl. Phys., 99, 084314.

Linavre, H. and H. Jordan (2009). Surface potential dependence of the Hamaker constant, Journal of physical chemistry, 113(11), 4419-4425.

Massaioli, F., R. Benzi and S. Succi (1993). Exponential tails in two-dimensional RayleighBe'nard convection. Europhys. Lett., 21, 305-310.

Nemati, H., M. Farhadi, K. Sedighi, E. Fattahi, A.A.R. Darzi (2010). Lattice Boltzmann simulation of nanofluid in lid-driven cavity. International Commun-ications in Heat and Mass Transfer, 37(10), 1528-1534.

Palmer, B. J. and D. R. Rector (2000). Lattice Boltzmann algorithm for simulating thermal flow in compressible fluids. Journal of Computational Phys., 161, 1-20.

Peng, Y., C. Shu and Y. T. Chew (2003). Simplified thermal lattice Boltzmann model for incompressible thermal flows. Phys. Rev., 68.

Rahmati, A. R. and M. Ashrafizaadeh (2009). A Generalized Lattice Boltzmann Method for ThreeDimensional Incompressible Fluid Flow Simulation. Journal of Applied Fluid Mechanics, 2(1), 71-95.
Razavi, S. E., J. Ghasemi, and A. Farzadi (2009). Flux modeling in the finite-volume Lattice Boltzmann approach. International Journal of Computational Fluid dynamics, 23(1), 69-77.

Shi, Y., T. S. Zhao, and Z. L. Guo (2004). Thermal lattice Bhatnagar-Gross-Krook model for flows with viscous heat dissipation in the incompressible limit. Phys. Rev.,70.

Shu, C., Y. Peng, and Y.T. Chew (2002). Simulation of natural convection in a square cavity by Taylor series expansion and least square-based lattice Boltzmann method. International Journal of Modern Physics, 13, 1399-1414.

Succi, S. (2001). The Lattice Boltzmann equation for fluid dynamics and beyond. New York, Oxford University Press Inc.

Shukla, R. K. and V. K. Dhir (2008). Effect of Brownian motion on thermal conductivity of nanofluids. Journal of Heat Transfer, 130(4), 1-12.

Ubertini, S., and S. Succi (2005). Recent advances of Lattice Boltzmann techniques on unstructured grids. Progress in Computational Fluid Dynamics, 5, 8596.

Van der Sman, R. G. M., (1997). Lattice Boltzmann scheme for natural convection in Porous media. International Journal of Modern. Phys., 8, 879-888.

Xuan, Y., K. Yu , and Q. Li (2005). Investigation on flow and heat transfer of nanofluids by the thermal Lattice Boltzmann model. Progress in Computational Fluid Dynamics, 5, 13-19.

Xuan, Y., and Z. Yao (2005). Lattice Boltzmann model for nanofluids. Heat and Mass Transfer, 41, 199205.

Yang, Y. T., and F. H. Lai (2011). Numerical study of flow and heat transfer characteristics of aluminawater nanofluids in a microchannel using the lattice Boltzmann method. International Communications in Heat and Mass Transfer, article in press. 